\title{
Daytime physical activity patterns and physical fitness in institutionalized elderly women: An exploratory study.
}

\section{$\operatorname{AUTHOR}(\mathrm{S}):$}

Ikezoe, Tome; Asakawa, Yasuyoshi; Shima, Hiroto; Kishibuchi, Kaoru; Ichihashi, Noriaki

\section{CITATION:}

Ikezoe, Tome ...[et al]. Daytime physical activity patterns and physical fitness in institutionalized elderly women: An exploratory study.. Archives of gerontology and geriatrics 2013, 57(2): 221-225

\section{ISSUE DATE:}

2013-09

URL:

http://hdl.handle.net/2433/175250

\section{RIGHT:}

(C) 2013 Elsevier Ireland Ltd.; This is not the published version. Please cite only the published version.; この論文は出版社版でありません。引 用の際には出版社版をご確認ご利用ください。 
Daytime physical activity patterns and physical fitness in institutionalized elderly women: An exploratory study

5 Tome Ikezoe ${ }^{1}$, Yasuyoshi Asakawa ${ }^{2}$, Hiroto Shima ${ }^{3}$, Kaoru Kishibuchi ${ }^{4}$,Noriaki Ichihashi ${ }^{1}$

1) Human Health Sciences, Graduate School of Medicine, Kyoto University, 53 Shogoin-Kawahara-cho, Sakyo-ku, Kyoto 606-8507, Japan

2) Course of Health Sciences, Graduate School of Medicine, Gunma University, Japan

10 3)Department of Rehabilitation, Uji Takeda Hospital, Japan

4)The Graduate School of Human \& Environmental Studies, Kyoto University, Japan

*Corresponding author

15 Tome Ikezoe

Telephone: +81-75-751-3967; fax: +81-75-751-3909

E-mail: ikezoe@hs.med.kyoto-u.ac.jp

20 Abstract

This study investigated the relationship between daytime physical activity patterns and physical fitness in elderly women. The subjects comprised 19 elderly women who resided in a nursing home. Time spent lying, sitting, standing and walking and the number of steps taken during the daytime from 10:00 to 16:00 were measured to determine physical activity patterns.

25 Physical fitness measures included muscle strength, balance, flexibility and physical performance. The elderly women spent $18.3 \%$ of their daytime walking, $7.31 \%$ in a standing position, $56.9 \%$ sitting and $17.4 \%$ lying down. Our results showed that the time spent in walking or standing positions was significantly associated with balance and physical performance such as walking speed, while the time spent in a sitting position was inversely

30 associated with muscle strength, balance and physical performance. The results of this study suggest that the time the elderly spend on weight-bearing activities and in sedentary behavior are associated with physical fitness.

\section{Key words}

35 Physical activity, Physical fitness, Elderly women, Sedentary behavior, Daytime activity patterns 


\section{Introduction}

Habitual physical activity decreases progressively with age (Meijer et al., 2001; Westerterp et al., 2001). The evidence clearly indicates that older people who are physically active have lower morbidity and mortality rates than inactive older people (Blair et al., 1989; Fried et al., 1998; Pate et al., 1995; Rohm-Young et al., 1995). A high habitual activity level appears to be a critical factor in improving or maintaining physical health.

The negative consequences of physical inactivity and sedentary behavior, such as prolonged sitting, in older people are also well established. Inactive lifestyles have been associated with mortality, decreased quality of life, chronic disease and health conditions such as cardiovascular disease, stroke, type 2 diabetes, obesity, some forms of cancer and depression (King AC et al., 1998, 2001; Owen N et al., 2012; Sundquist K et al., 2004). Therefore, there has been a growing acknowledgement of the importance of reducing sedentary activities as well as increasing physical activity in older age.

15 In addition, previous studies have demonstrated that physical activity is associated with physical function. Brach et al. (2004) showed that physical activity and exercise, assessed by a modified leisure-time physical activity questionnaire, were related to physical function in older persons, such as time to walk $400 \mathrm{~m}$ and knee extensor strength. Visser et al. (2005) showed that physical activity, assessed using an interviewer-administered questionnaire, had an effect on incident mobility limitation such as difficulty walking or climbing steps. Our previous study (Ikezoe et al., 2011) also found that daily physical activity assessed using life-space assessment (LSA) was positively correlated with the skeletal muscle mass of the lower extremity.

Thus, a number of studies have examined relationships between habitual physical activity and physical function. However, one limitation of these studies has been that physical activity patterns were assessed by questionnaires. Such subjective responses provide only limited estimates of habitual physical activity, particularly in older adults who commonly have difficulties in recall (Lagerros et al., 2007; Tehard et al., 2005) .

Walking step count measured by motion sensors such as pedometers and accelerometers is an objective and reliable way to assess the physical activity level even in older adults (Davis et al., 30 2007; Harris et al., 2009). Aoyagi et al. (2009) demonstrated that lower-extremity function such as walking speed and knee extension torque showed significant positive relationships with daily step count. Park et al. (2010) found that muscle mass in the lower limbs of older adults, as measured by whole-body dual X-ray absorptiometry, was associated with walking step count in daily life.

As well as increasing the number of walking steps taken per day, increasing the duration of weight-bearing activity such as walking and standing and decreasing inactive time in sitting and lying positions will be also critically important factors in preventing functional limitations in frail elderly people. However, to our knowledge, no investigations have examined the duration of activity types such as walking, standing, sitting and lying for habitual physical activity of daily living. The associations of these physical activity patterns with physical function in frail elderly people are also unclear. 
The aim of this study was to examine the time spent on each activity of daily living using motion sensors, to gain objective and accurate results, and to investigate the relationship between physical activity patterns and physical function in elderly women.

\section{Subjects and methods}

\subsection{Subjects}

The subjects comprised 19 elderly women, with a mean age of 83.8 years $(\mathrm{SD}=8.0$, range $=$ 71-96). All were residents of a nursing home in Kyoto, Japan, and therefore lived under the same conditions. The subjects were able to ambulate independently or with an assistive device, did not

10 have an unstable physical condition, and did not demonstrate severe dementia. Subjects with physical dysfunctions that may influence outcome measures, such as acute neurological impairment (acute stroke, Parkinson's disease, paresis of the lower limbs), severe musculoskeletal impairment and severe cognitive impairment, were excluded.

The subjects were informed about the study procedures before testing and provided written

15 informed consent before participating. The study was approved by Kyoto University Graduate School and Faculty of Medicine Ethics Committee.

\subsection{Physical activity assessment}

Physical activity pattern in the daytime was assessed objectively using the Activity Monitoring and Evaluation System (A-MES; Solid Brains Inc., Kumamoto, Japan), which consists of two three-dimensional acceleration sensors and technical software. The small sensors were attached to the anterior surface of the right thigh and the sternum by a custom-made fixation tape.

Three positions (lying, sitting, and standing) and walking were distinguished by the technical software using the data obtained from the sensors. Positions were estimated from the tilt direction for gravity by direct current composition of a sensor signal as follows: trunk sensor in a horizontal direction $=$ lying posture; trunk sensor in a vertical direction and thigh sensor in a horizontal direction $=$ sitting posture; trunk and thigh sensors in a vertical direction $=$ standing posture; both sensors in a vertical direction and large vertical accelerations periodically recorded $=$ walking. Time spent in each position and walking was recorded, and total time during the daytime was

30 calculated from the time data. The ratio of each position and walking in the daytime was calculated using the following equation: ratio in the daytime $(\%)=$ total time of each position and walking / measurement time $\times 100$.

The number of steps taken in the daytime was measured using an accelerometer (Yamasa Tokei Keiki Co., Ltd., Tokyo, Japan). The accelerometer was set superior to the iliac crest using a custom 35 pouch. Physical activity patterns assessed using accelerometers show that older people are less active in the morning and evening (from 1600) (Davis et al., 2007). Therefore, we assessed physical activity during the daytime from 1000 to 1600 hours. The participants were asked to maintain usual physical activity levels.

There is evidence of substantial seasonal changes in the physical activity patterns of the elderly 
2008). Changes in weather may also affect physical activity. Our data were collected for two days in autumn (from October to November) and winter (from December to January) under fine weather conditions such as when the sun was shining in a cloudless sky and average accelerometer step counts and time spent in different activity levels were obtained.

Although the A-MES device has previously been shown to be valid and reliable in the measurement of the time data for each position and for walking in elderly subjects (Kawagoshi et al., 2011), the validity and accuracy of our subjects was assessed in a pilot study. Ten healthy elderly women (mean age, 82.7 years; SD, 7.42) were recruited for the validity analysis. Time spent in each position and walking was continuously assessed for 30 minutes in a specific room using this device and visual observation by video monitoring synchronously. The accuracy of the measurements of each position and of walking was calculated using the following equation: Accuracy $(\%)=$ measurement time of this device / visual observation time $\times 100$. The accuracies for each position and for walking were 98.8-100.9 \%. Spearman's correlation coefficient was calculated to examine the relation between the measurement times of the device and the visual observation times. The results of the correlation coefficients for each position and for walking were $0.96-0.97(\mathrm{p}<0.01)$. These results indicated a high degree of validity and accuracy in measuring time spent on activities.

\subsection{Physical Fitness Measurements}

20 Physical fitness measures included muscle strength, balance, flexibility and physical performance.

\section{Muscle strength}

Quadriceps strength was used to represent muscle strength. Quadriceps strength of the right side was measured by a hand-held dynamometer ( $\mu$-Tas F-1, ANIMA Co., Tokyo, Japan) during a 3 second isometric contraction of the knee extensor. With the patient in a sitting position, the hip and knee were at $90^{\circ}$ angles and the force sensor was placed $10 \mathrm{~cm}$ above the lateral malleolus. The maximal isometric strength was determined as the larger of the two repeated measurements after pre-measurement trials with manual resistance. Torque $(\mathrm{Nm})$ was calculated by multiplying strength by the lever arm (distance between the lateral knee joint line and the point of force application).

\section{Balance test}

The one-legged stance test (OLST) with eyes open, postural sway in the standing position, and the stepping test in the standing position were used to measure balance function.

35 The OLST was performed in the standing position with the subject's arms by their sides. Timing was begun when the subjects raised one leg. Timing was stopped if the subjects moved the foot they were standing on, touched the suspended foot to the ground, or reached the maximum balance time of 120 seconds. Two trials were performed with the dominant foot if the maximum balance time was not reached on the first trial. The best balance time of the recorded trials was used for analysis. 
Postural sway was measured using a platform center of a foot pressure recorder (G-5500, ANIMA Co., Tokyo, Japan) for 20 seconds. The subject's arms were held alongside the body, with the feet parallel to each other. No assistive devices were allowed and their eyes were open. The length of the displacement of the center of gravity (LNG; $\mathrm{cm}$ ) was used as the body sway parameter.

The stepping test (Ikezoe et al., 2009) in a standing position was used to measure dynamic balance function. The subject was asked to step as rapidly as possible, and the total number of steps within 5 seconds was measured using a step counter (Takei Co., Nigata, Japan). The best count of two trials was recorded.

10

\section{Flexibility test}

A sit and reach test was used to measure flexibility. The subjects were seated with their knee joints fully extended and their hip joints flexed at about $90^{\circ}$ to assume an upright sitting position. They were then asked to bend forward slowly and reach forward as far as possible from this seated

15 position. The score was determined as the distance the subject was able to reach with the fingertips on a scale in centimeters.

\section{Performance test}

The maximal walking test (MWT), the timed "Up \& Go" test (TUG) (Podsiadlo et al., 1991) and the chair stand test (Whitney et al., 2005) were used to determine physical performance.

MWT was determined over a $5 \mathrm{~m}$ distance. The subject was asked to walk as fast as possible. The walking time of the $5 \mathrm{~m}$ distance was recorded using a stopwatch, and walking speed $(\mathrm{m} / \mathrm{s})$ was calculated.

For the TUG, the subject was instructed to stand up from a sitting position in an armless chair, walk a distance of $3 \mathrm{~m}$, turn, walk back to the chair, and sit down. The test was performed once at maximum walking speed after each subject performed one trial to become familiar with the test. The time was measured from the start until the subject sat down in the chair.

The participants wore ordinary shoes and walking aids were permitted during TUG and MWT.

The chair stand test measured the time needed to stand five times from a standard chair. Subjects 30 were asked to rise to a full standing position and then return back to a seated position as fast as possible. Timing began when the examiner said "Go" and stopped when the subject's buttocks touched the chair on the fifth repetition. The chairs used were all $46 \mathrm{~cm}$ high, armless and had slightly padded seats.

\subsection{Statistical analyses}

All data are presented as mean \pm SD.

Spearman's correlation coefficient was used to investigate the relationships between number of steps and time spent on activities in elderly persons. Spearman's correlation coefficient was used to investigate the relationship between the physical activity measurements and physical fitness. 
was recognized at $\mathrm{p}<0.05$.

\section{Results}

\subsection{Physical activity level and physical fitness in the elderly}

Tables 1 and 2 show physical activity level and physical fitness, respectively (mean and SD). The ratios of specific positions and walking in the daytime (from 1000 to 1600 hours) were: walking $18.3 \%$ (SD 8.60); standing $7.31 \%$ (SD 6.40); sitting $56.9 \%$ (SD 19.8); and lying $17.4 \%$ (SD 18.7). Older subjects spent the most time in the sitting position.

\subsection{Relationships between number of steps and time spent on activities}

Table 3 shows the relationships between number of steps and time spent on activities. Correlation analysis revealed that significant relationships were not observed between number of steps and time spent on activities.

\subsection{Relationship between physical activities and physical fitness}

Table 4 shows the relationship between physical activities and physical fitness. Quadriceps strength was significantly and positively associated with number of steps, whereas a significant negative correlation was found between quadriceps strength and total time sitting. OLST was negatively correlated with total time sitting. LNG showed a significant association only with total time standing. Stepping was positively correlated with total time walking and number of steps, but was negatively correlated with total time sitting. There was no significant relationship between the sit and reach test and physical activities. MWT and TUG were related to total time walking and number of steps, but were inversely related to total time sitting. The chair stand test showed a significant association only with number of steps.

\section{Discussion}

There are many beneficial effects of increased physical activity in preventing further functional disability (Boyle et al., 2007; Christensen et al., 2006; Haveman-Nies et al., 2003; Schroll et al., 1997; Simoes et al., 2006) and maintaining public health (Haskell et al., 2007; Pate et al., 1995;

30 Yasunaga et al., 2006) among elderly subjects. Another important concept is the limitation of sedentary behavior. The sedentary time represents the duration of occupations that expend very little energy above resting metabolic rate, e.g., sitting. Sedentary behavior is also a risk factor for poor physical health (Bertrais et al., 2005; Hamilton et al., 2007; Hu et al., 2003). Therefore, elderly people wanting to increase their activity levels should be recommended to spend less time sitting or lying, and more time walking or standing (Pate et al., 1995).

A number of studies have examined relationships between habitual physical activity, assessed using a self-reported questionnaire or daily step count, and physical function in community-based elderly people with relatively high activity levels. However, there are no data showing habitual physical activity of daily living measured objectively and distinguishing between different activity levels such as walking, standing, sitting and lying positions in frail, institutionalized elderly people. 
To our knowledge, ours is the first study to investigate this. In addition, this is the first report showing the relationship between physical activity patterns and physical function.

Our analyses of the relationships between number of steps and time spent on activities indicated that not all of the correlations were significant. The results suggest that the total time spent on each activity of daily living during the daytime was not related to number of steps in frail elderly people.

Although our data represent only 6 hours during the daytime, the institutionalized elderly women in our study were taking about 2000 steps and spending 66 minutes walking. The step count was limited to about 2000 steps, which may also have been influenced by a generally lower walking speed in our institutionalized elderly subjects compared to community-based elderly people (Bohannon, 1997). Jacobi et al. (2009) showed the importance of assessing sedentary time among physical activity domains in older adults with low habitual physical activity levels. Our findings showed that the elderly women spent $56.9 \%$ of their daytime sitting and $17.4 \%$ lying down. This suggests that at least in this population, elderly women spend much sedentary and inactive time even during daylight hours.

15 Our analyses of the relationships between physical activities and physical fitness indicated that number of steps was associated with quadriceps strength, but not with LNG or flexibility. This is in accordance with a previous study (Aoyagi et al., 2009) that showed a significant positive relationship between daily step count and knee extension torque but not body sway. A lack of muscle overload resulting from a reduced number of steps may increase the risk of sarcopenia. Park

20 et al. (2010) demonstrated that seniors who walked at least 7000-8000 steps/day and/or spent 15-20 min/day at an intensity of > 3 metabolic equivalents (METs) were likely to have a muscle mass above the sarcopenia threshold. Our findings suggested that frail older adults may be encouraged to engage regularly in higher impact weight bearing activity such as long-distance walking to maintain their muscle strength. Conversely, increasing muscle strength may have a greater impact on maintaining or improving walking endurance.

Total times spent walking and standing were associated with balance function, but not with quadriceps strength. The results indicated that if elderly women were able to engage in high levels of activity such as walking or in a standing position for a longer time, even if the activities were not continued, this may have an effect on balance function, while long-distance walking may be related to muscle strength in their lower extremities.

Another important concept is sedentary time in elderly women. The present study showed that quadriceps strength, OLST, stepping, MWT and TUG were inversely related to the time spent in sitting activities. Engaging in much sedentary time may lead to a decline in physical fitness such as muscle strength, balance and functional ability. In addition, decreases in physical fitness, particularly muscle strength, balance and physical performance, may be a risk factor for sedentary behavior among elderly women.

There were no significant associations between the time spent in a lying position and physical fitness. Some elderly subjects spent a long time in a lying position because they have a habit of taking a nap. Therefore, the time spent lying down may not have been related to physical fitness. It also seems likely that various aspects aside from physical fitness, such as chronic health conditions 
and mental and psychosocial health, influence the time spent in a lying position.

Annually, an increasing number of studies have continued to document the benefits of physical activity for elderly people, but most of these studies have focused on "healthy, active elderly" subjects. Our study focused on the residents of a nursing home because of the high prevalence of physical frailty and functional dependence among nursing home residents as a result of physical inactivity and acceptance of a sedentary lifestyle. The findings of this study suggest that physical inactivity is strongly associated with functional decline in frail nursing home residents with lower physical fitness than that found in community-dwelling elderly. A sedentary lifestyle can have a devastating effect on the individual's independence, quality of life, morbidity and mortality, often leading to a spiral of inactivity and further decline. Therefore, clinical care and practice to promote physical activity in long term care settings should target decreasing sedentary time as well as increasing walking or standing time in the frail elderly population.

This study had some limitations, including the small number of subjects. The stringent exclusion criteria, which limited the number of subjects, may also limit the generalizability of the findings.

15 The other limitation arose from the cross-sectional structure of this study. For the sedentary behavior in the elderly women, the causality was not clear, i.e., whether poor physical fitness resulted from or led to sedentary behavior. We need to conduct further research, using longitudinal designs, to validate the positive effects of physical activity and the negative consequences of inactivity, including multidimensional factors such as morbidity and mortality outcomes.

\section{Conclusions}

Institutionalized elderly women spend much time in sedentary, inactive positions such as sitting and lying even in the daytime. Our findings showed that the time spent on walking and standing activities was related to balance and physical performance, while sedentary time was inversely related to muscle strength, balance and physical performance. This study suggests that physical activity patterns in frail elderly people are associated with many aspects of physical fitness, including muscle strength, balance and functional ability, and that it is necessary to decrease sedentary time as well as to increase walking or standing time among frail nursing home residents.

Conflict of interest statement: None.

\section{References}

Aoyagi, Y., \& Shephard, R.J. (2009). Steps per day: the road to senior health? Sports medicine, 39, 423-438.

Aoyagi, Y., Park, H., Watanabe, E., Park. S., \& Shephard, R.J. (2009). Habitual physical activity and physical fitness in older Japanese adults: the Nakanojo Study. Gerontology, 55, 523-531.

Bertrais, S., Beyeme-Ondoua, J.P., Czernichow, S., Galan, P., Hercberg, S., \& Oppert, J.M. (2005). Sedentary behaviors, physical activity, and metabolic syndrome in middle-aged French subjects. Obes Res, 13, 936-944. 
Blair, S.N., Kohl, H.W., Paffenbarger, R.S. Jr., Clark, D.G., Cooper, K.H., \& Gibbons, L.W. (1989). Physical fitness and all-cause mortality: A prospective study of healthy men and women. Journal of the American Medical Association, 262, 2395-2401.

Bohannon, R.W. (1997). Comfortable and maximum walking speed of adults aged 20-79 years: reference values and determinants. Age and Ageing, 26, 15-19.

Boyle, P., Buchman, A.S., Wilson, R., Bienias, J.L., \& Bennett, D. (2007). Physical activity is associated with incident disability in community-based older persons. Journal of the American Geriatrics Society, 55, 195-201.

Brach, J.S., Simonsick, E.M., Kritchevsky, S., Yaffe, K., \& Newman, A.B. Health, Aging and Body Composition Study Research Group (2004). The association between physical function and lifestyle activity and exercise in the health, aging and body composition study. Journal of the American Geriatrics Society, 52, 502-509.

Christensen, U., Stovring, N., Schultz-Larsen, K., Schroll, M., \& Avlund, K. (2006). Functional ability at age 75: is there an impact of physical inactivity from middle age to early old age? Scandinavian Journal of Medicine and Science in Sports, 16, 245-251.

Davis, M.G., \& Fox, K.R. (2007). Physical activity patterns assessed by accelerometry in older people. European Journal of Applied Physiology, 100, 581-589.

Fried, L.P., Kronmal, R.A., Newman, A.B., Bild, D.E., Mittelmark, M.B., Polak, J.F., et al. (1998). Risk factors for 5-year mortality in older adults: the Cardiovascular Health Study. Journal of the American Medical Association, 279, 585-592.

Hamilton, M.T., Hamilton, D.G., \& Zderic, T.W. (2007). Role of low energy expenditure and sitting in obesity, metabolic syndrome, type 2 diabetes, and cardiovascular disease. Diabetes, 56. 2655-2667.

Harris, T.J., Owen, C.G., Victor, C.R., Adams, R., Ekelund, U., \& Cook, D.G. (2009). A comparison of questionnaire, accelerometer, and pedometer: measures in older people. Medicine and Science in Sports and Exercise, 41, 1392-1402.

Haskell, W.L., Lee, I.M., Pate, R.R., Powell, K.E., Blair, S.N., Franklin, B.A., et al. (2007) .Physical activity and public health: updated recommendation for adults from the American college of sports medicine and the American heart association. Medicine and Science in Sports and Exercise, 39, 1423-1434.

Haveman-Nies, A., de Groot, L., \& van Staveren, W. (2003). Relation of dietary quality, physical activity, and smoking habits to 10-year changes in health status in older Europeans in the SENECA study. American Journal of Public Health, 93, 318-323.

Hu, F.B., Li, T.Y., Colditz, G.A., Willett, W.C., \& Manson, J.E. (2003). Television watching and other sedentary behaviors in relation to risk of obesity and type 2 diabetes mellitus in women. Journal of the American Medical Association, 289, 1785-1791.

Ikezoe, T., Asakawa, Y., Shima, H., \& Ichihashi, N. (2009). Physical function screening of institutionalized elderly women to predict their risk of falling. Journal of Physical Fitness and Sports Medicine, 58, 489-498.

40 Ikezoe, T., Mori, N., Nakamura, M., \& Ichihashi, N. (2011). Age-related muscle atrophy in the 
lower extremities and daily physical activity in elderly women. Archives of Gerontology and Geriatrics, 53, e153-157.

Jacobi, D., Charles, M.A., Tafflet, M., Lommez, A., Borys, J.M., \& Oppert, J.M. (2009). Relationships of self-reported physical activity domains with accelerometry recordings in French adults. European Journal of Epidemiology, 24, 171-179.

Kawagoshi, A., kiyokawa, N., Sugawara, K., Takahashi, H., Abe, R., Kitamura, N., Satake, M., \& Shioya, T. (2011). The quantitative assessment of the physical activity of daily life in patients with stable elderly COPD using an Activity Monitoring and Evaluation System. J Jpn Phys Ther Assoc, 38, 497-504.

King, A.C., Rejeski, W.J., \& Buchner, D.M. (1998). Physical activity interventions targeting older adults. A critical review and recommendations. Am J Prev Med, 15, 316-333.

King, A.C. (2001). Interventions to promote physical activity by older adults. J Gerontol A Biol Sci Med Sci, 56, 36-46.

Lagerros, Y.T., Lagiou, P. (2007). Assessment of physical activity and energy expenditure in epidemiological research of chronic diseases. European Journal of Epidemiology, 22, 353-362.

Meijer, E.P., Goris, A.H., Wouters, L., \& Westerterp, K.R. (2001). Physical inactivity as a determinant of the physical activity level in the elderly. International Journal of Obesity and Related Metabolic Disorders, 25, 935-939.

Owen, N. (2012). Sedentary behavior: understanding and influencing adults' prolonged sitting time. Prev Med, 55, 535-539.

Park, H., Park, S., Shephard, R.J., \& Aoyagi, Y. (2010). Yearlong physical activity and sarcopenia in older adults: the Nakanojo Study. European Journal of Applied Physiology, 109, 953-961.

Pate, R.R., Pratt, M., Blair, S.N., Haskell, W.L., Macera, C.A., Bouchard, C., et al. (1995). Physical activity and public health: A recommendation from the Centers for Disease Control and Prevention and the American College of Sports Medicine. Journal of the American Medical Association, 273, 402-407.

Podsiadlo, D., \& Richardson, S. (1991). The timed "Up \& Go": a test of basic functional mobility for frail elderly persons. Journal of the American Geriatrics Society, 39, 142-148.

Rohm-Young, D., \& Masaki, K.H. (1995). Associations of physical activity with performance-based and self-reported physical functioning in the older men: The Honolulu Heart Program. Journal of the American Geriatrics Society ,43, 845-854.

Schroll, M., Avlund, K., \& Davidsen, M. (1997). Predictors of five-year functional ability in a longitudinal survey of men and women aged 75 to 80. The 1914- population in Glostrup, Denmark. Aging (Milano), 9, 143-152.

Shephard, R.J., \& Aoyagi, Y. (2009). Seasonal variations in physical activity and implications for human health. European Journal of Applied Physiology ,107, 251-271.

Simoes, E., Kobau, R., Kapp, J., Waterman, B., Mokdad, A., \& Anderson, L. (2006). Associations of physical activity and body mass index with activities of daily living in older adults. Journal of Community Health, 12, 115-121. 
Sundquist, K., Qvist, J., Sundquist, J., \& Johansson, S.E. (2004). Frequent and occasional physical activity in the elderly: a 12-year follow-up study of mortality. Am J Prev Med, 27, 22-27.

Tehard, B., Saris, W.H., Astrup, A., Martinez, J.A., Taylor, M.A., Barbe, P., et al. (2005) .Comparison of two physical activity questionnaires in obese subjects: the NUGENOB study. Medicine and Science in Sports and Exercise, 37, 1535-1541.

Togo, F., Watanabe, E., Park, H., Shephard, R.J., \& Aoyagi, Y. (2005). Meteorology and the physical activity of the elderly: the Nakanojo Study. International Journal of Biometeorology, 50, 83-89.

Togo, F., Watanabe, E., Park, H., Yasunaga, A., Park, S., Shephard, R.J., \& Aoyagi, Y. (2008). How many days of pedometer use predict the annual activity of the elderly reliably? Medicine and Science in Sports and Exercise, 40, 1058-1064.

Visser, M., Simonsick, E.M., Colbert, L.H., Brach, J., Rubin, S.M., Kritchevsky, S.B., et al.; for the Health ABC Study (2005). Type and intensity of activity and risk of mobility limitation: the mediating role of muscle parameters. Journal of the American Geriatrics Society, 53, 762-770.

15 Whitney, S.L., Wrisley, D.M., Marchetti, G.F., Gee, M.A., Redfern, M.S., \& Furman, J.M. (2005). Clinical measurement of sit-to-stand performance in people with balance disorders: validity of data for the Five-Times-Sit-to-Stand Test. Physical Therapy, 85, 1034-1045.

Yasunaga, A., Togo, F., Watanabe, E., Park, H., Shephard, R.J., \& Aoyagi, Y. (2006). Yearlong physical activity and health-related quality of life in older Japanese adults: the Nakanojo Study. Journal of Aging and Physical Activity, 14, 288-301. 


\section{Table 1}

Time spent on activities and number of steps in the elderly $(n=19)$

5

\begin{tabular}{lr}
\hline Total time (minutes) & \\
walking & $66.0 \pm 31.0$ \\
standing & $26.3 \pm 23.1$ \\
sitting & $205.2 \pm 71.6$ \\
lying & $62.7 \pm 67.5$
\end{tabular}

Ratio in the daytime (\%)

$\begin{array}{lr}\text { walking } & 18.3 \pm 8.60 \\ \text { standing } & 7.31 \pm 6.40 \\ \text { sitting } & 56.9 \pm 19.8 \\ \text { lying } & 17.4 \pm 18.7 \\ \text { ber of steps (steps) } & 2004.8 \pm 1998\end{array}$

Ratio in the daytime $(\%)=$ total time of each position and walking / measurement time $\times 100$

Values are expressed as mean \pm standard deviation (SD)

Table 2

Results of physical fitness $(n=19)$

\begin{tabular}{ll}
\hline Quadriceps strength $(\mathrm{Nm})$ & $44.4 \pm 19.3$ \\
OLST $(\mathrm{s})$ & $9.92 \pm 16.3$ \\
LNG $(\mathrm{cm})$ & $45.2 \pm 17.6$ \\
Stepping (steps) & $23.1 \pm 9.06$ \\
Sit and reach (cm) & $25.8 \pm 7.89$ \\
MWT (m/s) & $1.10 \pm 0.34$ \\
TUG (s) & $9.86 \pm 3.26$ \\
chair stand (s) & $10.4 \pm 4.10$ \\
\hline
\end{tabular}

15

Values are expressed as mean \pm standard deviation (SD) 
Table 3

Relationships between number of steps and time spent on activities $(n=19)$

\begin{tabular}{ccl}
\hline & $\begin{array}{c}\text { correlation } \\
\text { coefficients }\end{array}$ & $\mathrm{p}$ value \\
\hline Total time & & \\
walking & 0.43 & 0.07 \\
standing & 0.19 & 0.43 \\
sitting & -0.37 & 0.12 \\
lying & 0.12 & 0.62 \\
\hline
\end{tabular}

Table 4

Relationship between physical activities and physical fitness $(n=19)$

15

\begin{tabular}{ccccccccc}
\hline & $\begin{array}{c}\text { Quadriceps } \\
\text { strength }\end{array}$ & OLST & LNG & Stepping & $\begin{array}{c}\text { Sit and } \\
\text { reach }\end{array}$ & MWT & TUG & $\begin{array}{c}\text { Chair } \\
\text { stand }\end{array}$ \\
\hline Total time & & & & & & & & \\
walking & 0.15 & 0.39 & -0.18 & $0.53^{*}$ & -0.04 & $0.63 * *$ & $-0.57^{*}$ & -0.40 \\
$\quad$ standing & 0.22 & 0.35 & $-0.48^{*}$ & 0.10 & 0.28 & -0.03 & -0.09 & -0.32 \\
sitting & $-0.50^{*}$ & $-0.51^{*}$ & 0.17 & $-0.61^{* *}$ & 0.25 & $-0.59^{* *}$ & $0.52^{*}$ & 0.38 \\
$\quad$ lying & 0.11 & 0.23 & -0.07 & 0.38 & -0.21 & -0.36 & -0.32 & -0.30 \\
Number of steps & $0.46^{*}$ & 0.38 & 0.01 & $0.46^{*}$ & -0.00 & $0.54^{*}$ & $-0.48^{*}$ & $-0.50^{*}$
\end{tabular}

Values expressed are correlation coefficients

${ }^{* *}, \mathrm{p}<0.01 ;{ }^{*}, \mathrm{p}<0.05$ significant correlation 